\title{
Data Separation of $\ell_{1}$-minimization for Real-time Motion Detection
}

Yu Liu ${ }^{1}$

jasonyuliu@nudt.edu.cn

Huaxin Xiao ${ }^{1}$

xiaohuaxin@nudt.edu.cn

Zheng Zhang ${ }^{1}$

zhangzheng@nudt.edu.cn

Wei $\mathrm{Xu}^{1}$

xuwei@nudt.edu.cn

Maojun Zhang ${ }^{1}$

mjzhang@nudt.edu.cn

Jianguo Zhang ${ }^{2}$

jgzhang@computing.dundee.ac.uk
${ }^{1}$ College of Information System and Mangement

National University of Defense Technology

Changsha, China

${ }^{2}$ School of Computing

University of Dundee

Dundee DD1 4HN, U.K.
The $\ell_{1}$-minimization $\left(\ell_{1}\right.$-min) used to seek the sparse solution restricts the applicability of compressed sensing. In this study, We use the existing $\ell_{1}$-min algorithms as a pre-process step that converts the iterative optimization into linear addition and multiplication operations. This paper then proposes a data separation algorithm with computationally efficient strategies to achieve real-time performance of sparse-based motion detection.

For a given signal $y \in \mathbb{R}^{m}$, the proposed approach separates $y$ as the linear combination of the basis vectors $e_{i}$, which is defined as atoms in this paper:

$$
y=\gamma_{1} e_{1}+\cdots+\gamma_{i} e_{i}+\cdots \gamma_{m} e_{m},
$$

where $\gamma_{i}$ is the coefficient of $y$ over the basis vectors $e_{i}$, and it could be computed easily by simply projecting $y$ on a basic vector $e_{i}$, i.e., the $i$-th coordinate of $y$. For example, the image vector in this work is separated to the smallest atoms $e_{i}$ which contains one non-zero standard element as:

$$
e_{i}=\left[0,0, \cdots,{ }_{i}, \cdots, 0,0\right]^{T} .
$$

The signal is not limited to the atom used in this work, but can be separated into a variety of patterns of atoms. Each $e_{i}$ can be considered as the observed signal and convert original $\ell_{1}$-min problem $P_{1}$ as follows:

$$
P_{1}^{e_{i}}: \quad \hat{\boldsymbol{\beta}}_{i}=\arg \min \left\|\boldsymbol{\beta}_{i}\right\|_{1} \quad \text { s.j. } D \boldsymbol{\beta}_{i}=e_{i},
$$

where $\boldsymbol{\beta}_{i}$ is defined as the children sparse vector in this paper.

Inspired by [2], we assume that the sparse solution $\alpha$ of $y$ can be separated into the linear combination of its children sparse vector $\boldsymbol{\beta}_{i}$ as follows:

$$
\alpha \approx \gamma_{1} \boldsymbol{\beta}_{1}+\cdots+\gamma_{i} \boldsymbol{\beta}_{i}+\cdots \gamma_{m} \boldsymbol{\beta}_{m} .
$$

For the motion detection problem, we can pre-solve the children sparse vector $\boldsymbol{\beta}_{i}$ with the existing $\ell_{1}$-min algorithms. Then, the sparse solution of a new signal or image $y$ can be rapidly obtained by Eq. (4). Then, the iterative process in the existing $\ell_{1}$ algorithms is replaced by addition and multiplication operations.

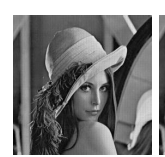

(a)

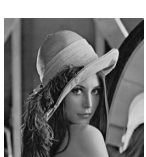

(b)

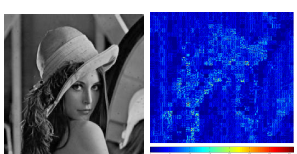

(c)

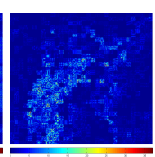

(e)

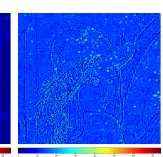

(f)
Figure 1: (a)-(c): The recovered Lena image $(256 \times 256)$ by proposed Data separation, Bregman iterative [3], and Lasso [1]. Their execution time of recovery are $0.12 \mathrm{~s}, 100.16 \mathrm{~s}$, and $4.18 \mathrm{~s}$, respectively. (d)-(e): The recovered error by proposed Data separation, Bregman iterative [3], and Lasso [1]. The percentage of recovered error in pixel level is 0.0331 , 0.0176 and 0.1317 respectively.

Another important question is about the numerical distance of the sparse solution between the use of the classic $\ell_{1}$-min and data separation algorithm. The distance is acceptable for many applications, (e.g., motion detection), but not for others, (e.g., image deblurring). If tolerable in a specific work, distance can be used as acceleration engine, which can dramatically improve applicability. The numerical distance can be visually represented as the recovered error in Fig. 1. Although the recovered results are not the best, the proposed data separation approach can significantly accelerate the $\ell_{1}$-min.

We construct the image atoms $e_{i}$ in Eq. (2) as the same size as the background $I_{B}$. The Bregman iterative algorithm [3] is employed to calculate the children sparse vector $\boldsymbol{\beta}_{i}$ of $e_{i}$. Consequently, the background model based on sparse representation can be rewritten as follows:

$$
I_{B}=D \times \alpha \approx D \times \sum_{i} \gamma_{i} \boldsymbol{\beta}_{i},
$$

where $\gamma_{i}$ is linear coefficients of the background model $I_{B}$ over the atom $e_{i}$.

Detection requires a high level of consideration to enhance the robustness because the dynamic textures or complicated environment can affect the corrupted frame $I$. In this work, we project the current frame $I$ over the pre-learned dictionary $D$ and compute the sparse codes $\alpha^{\prime}$ with the data separation algorithm. Then, the formula is converted as follows:

$$
\begin{aligned}
P_{F}^{i} & =D \alpha^{\prime}-D \alpha \approx D \times \sum_{j} \gamma_{j}^{\prime i} \boldsymbol{\beta}_{j}-D \times \sum_{j} \gamma_{j}^{i} \boldsymbol{\beta}_{j} \\
& =D \times \sum_{j}\left(\gamma_{j}^{i}-\gamma_{j}^{i}\right) \boldsymbol{\beta}_{j},
\end{aligned}
$$

where $\gamma_{i}^{\prime}$ is linear coefficients of current frame $I$ over the atom $e_{i}$.

Accordingly, we compare each patch to decide whether the frame belongs to the foreground through the distribution of the sparse coefficients.

$$
\left\{\begin{array}{l}
\Delta_{1}=\left\|\sum_{i} \gamma_{i}^{\prime} \beta_{i}-\sum_{i} \gamma_{i} \beta_{i}\right\|_{1}, \\
\Delta_{2}=\left|\left\|\sum_{i} \gamma_{i}^{\prime} \beta_{i}\right\|_{0}-\left\|\sum_{i} \gamma_{i} \beta_{i}\right\|_{0}\right|,
\end{array}\right.
$$

where $\Delta_{1}$ and $\Delta_{2}$ are the differences of sparse coefficients distributions and values between the background model and the current frame.

To obtain a more precision result, we post-process the differences of the sparse coefficients as shown in follow:

$$
\Delta=\lambda_{1} \Delta_{1}+\lambda_{2} \Delta_{2}
$$

where $\lambda_{1}$ and $\lambda_{2}$ are the unitary parameters which determine the weight of $\Delta_{1}$ and $\Delta_{2}$ respectively.

This work is at very preliminary stages. How the signal separated into basic atoms has remained an open question. A satisfactory result can be obtained in separating the signal even with the use of the simplest method as demonstrated by this work. Another future work is to measure the numerical difference of the sparse solution with or without using data separation. The difference is acceptable for motion detection, but it does not mean it can be used for other applications. Thus, mathematically defining this difference is able to decide the potential of the data separation algorithm.

[1] B. Efron, T. Hastie, I. Johnstone, and R. Tibshirani. Least angle regression. The Annals of statistics, 32(2):407-499, 2004.

[2] Y. C. Eldar and G. Kutyniok (Eds.). Compressed sensing: theory and applications. Chapter 11. Cambridge University Press, ISBN: 9781107005587, 2012.

[3] W. Yin, S. Osher, D. Goldfarb, and J. Darbon. Bregman iterative algorithms for compressed sensingand related problems. SIAM J. Imaging Sciences, 1(1):143-168, 2008. 